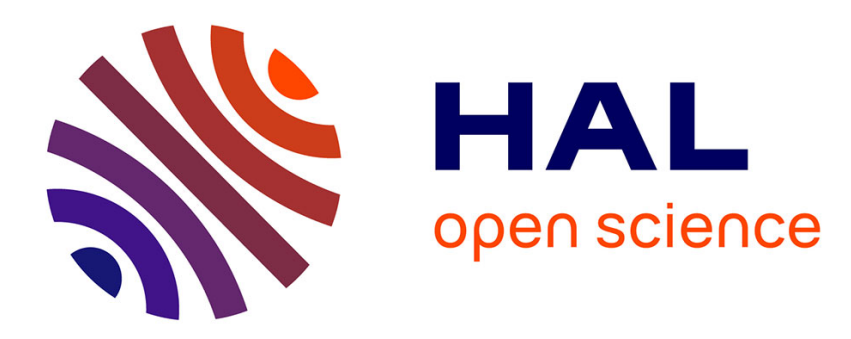

\title{
L'espace politique aux Antilles françaises
}

Justin Daniel

\section{To cite this version:}

Justin Daniel. L'espace politique aux Antilles françaises. Ethnologie française, 2002, 32 (4), pp.589600. 10.3917/ethn.024.0589 . hal-01675660

\section{HAL Id: hal-01675660 https://hal.univ-antilles.fr/hal-01675660}

Submitted on 11 Jan 2018

HAL is a multi-disciplinary open access archive for the deposit and dissemination of scientific research documents, whether they are published or not. The documents may come from teaching and research institutions in France or abroad, or from public or private research centers.
L'archive ouverte pluridisciplinaire HAL, est destinée au dépôt et à la diffusion de documents scientifiques de niveau recherche, publiés ou non, émanant des établissements d'enseignement et de recherche français ou étrangers, des laboratoires publics ou privés. 


\title{
L'ESPACE POLITIQUE AUX ANTILLES FRANCAISES
}

\author{
Justin DANIEL \\ Professeur de science politique \\ Université des Antilles françaises \\ UMR-CNRE 8053 LC2S \\ d.justin@orange.fr
}

\begin{abstract}
Résumé
Après avoir brièvement abordé le rôle des clivages nés de la subordination coloniale dans la formation des identités aux Antilles françaises, l'article s'intéresse plus particulièrement au passage au politique des revendications identitaires. L'accent est mis sur deux modalités du processus - la territorialisation des structures partisanes et la mobilisation des ressources locales - tout en insistant sur la dimension stratégique des proclamations identitaires et les enjeux de pouvoir qui les guident. Enfin un développement particulier est consacré aux contradictions de la démarche nationaliste : les tentatives de superposition de l'identité culturelle et de l'identité politique sont contrariées par l'insertion des acteurs sociaux dans une pluralité d'espace sociaux et culturels ainsi que la multiplicité des allégeances et des niveaux d'identification.
\end{abstract}

Mots clés : Identités, Culture, Politique, Pouvoirs locaux, nationalisme.

\begin{abstract}
The political space in the French West Indies

After a brief examination of the cleavages resulting from colonial subordination in the French West Indies, the paper analyzes the transition from identity claims to political claims and more especially two modalities of the process: the territorialization of partisan structures and the mobilization of local resources. Contradictions involved in the nationalist approach are also studied: attempts at superimposing cultural and political identities are thwarted by the insertion of social actors into a plurality of social and cultural spaces as well as by the multiplicity of allegiances and identification levels.
\end{abstract}

Keywords: Culture. Politics. Local Power. Nationalism. 
L'étude des stratégies identitaires qui se déploient dans le champ politique aux Antilles françaises est exemplaire à plus d'un titre. La trajectoire suivie par ces territoires semble en effet ruser avec l'histoire de la décolonisation. La voie empruntée est pour le moins hétérodoxe au regard des expériences de décolonisation observées ailleurs : elle débouche sur l'intégration formelle à un ensemble plus vaste et sur un jeu complexe d'appartenances juridico-institutionnelles imbriquées qui influent directement sur la formation des identités. Du même coup, se trouvent confirmés, de manière pour le moins éclatante, les acquis de l'anthropologie qui a depuis longtemps attiré l'attention sur le morcellement des identités, les processus de recomposition permanente qui les déterminent, et le caractère problématique de la synthèse qui en découle [Lévi-Strauss, 1977]. Constat sans doute banal aujourd'hui, mais qui emporte une conséquence dont l'analyse pourra difficilement se départir en la circonstance: l'identité est par définition une construction évolutive fortement dépendante des stratégies d'acteurs insérés dans une pluralité d'espaces sociaux et culturels.

En outre, dans un contexte où le passé colonial chevauche la réalité présente de l'Etat providence, où les allégeances se démultiplient au gré d'influences internes et externes, les revendications portant sur le statut politique tendent à structurer le jeu partisan. Elles paraissent défier, dans les solutions qu'elles présupposent, les catégories juridico-politiques classiques, soulignant au passage l'originalité et la complexité de la situation. L'appartenance à un ensemble plus vaste va ainsi de pair 
avec l'énonciation quasi-permanente de «différences»: les sociétés antillaises nourrissent en leur sein, tantôt sous une forme atténuée, tantôt sur un mode vif, toujours en fonction de stratégies fortement contextualisées, un débat récurrent sur le thème du lien séculaire les unissant à la France. Il en résulte que la question identitaire qui recouvre de nombreux usages sociaux et politiques mobilise et constitue l'un des répertoires privilégiés d'énonciation du politique.

Ces phénomènes semblent avoir connu une amplification récente. Mais la réactivation des revendications statutaires et l'apparente radicalisation des mobilisations identitaires ne doivent pas conduire à une erreur d'interprétation. Si elles paraissent en parfaite congruence avec des tendances constatées ailleurs, elles ne sauraient pour autant être appréhendées comme la simple résultante de dynamiques externes globales. Celles-ci sont sans aucun doute à l'œuvre : il suffit de rappeler les inquiétudes et les tentations d'un repli sur soi qui ont accompagné, aussi bien en Guadeloupe qu'à la Martinique, la mise en place du marché unique européen. Mais encore faut-il les articuler aux dynamiques internes - s'inscrivant nécessairement dans une historicité propre - des deux sociétés et prendre en compte les effets ainsi générés dans les espaces politiques insulaires. C'est là un passage obligé pour tenter de comprendre pourquoi et comment l'identité fait sens et acquiert, par les usages sociaux et les significations auxquels elle se prête, un caractère performatif lui conférant la force d'une réalité entrant en résonance directe avec le politique. 
Partant de ce constat, cette brève analyse propose, sans aucune prétention à l'exhaustivité, de rappeler quelques traits saillants des processus de construction identitaire aux Antilles françaises. Les clivages nés de la subordination coloniale ont durablement structuré les représentations de l'identité et ont suscité en retour des stratégies défensives. Mais le passage au politique de la revendication identitaire, notamment sous les différentes formes empruntées au cours de ces dernières années, n'est pas la conséquence aveugle et exclusive des tentatives d'imposition d'une mono-identification de la part de l'Etat. Il est tout autant le produit d'un travail de construction opéré localement par les élites aptes à maîtriser le récit identitaire, à le formaliser et à l'utiliser. D'où, la nécessité de procéder, dans un second temps, à l'examen de la place et du rôle des affirmations identitaires dans les stratégies structurant les espaces politiques insulaires. De ce point de vue, un rapide examen de la situation révèle que les enjeux de pouvoir, souvent médiatisés par des discours à connotation culturaliste, sont presque toujours au fondement des proclamations et des revendications identitaires, qu'il s'agisse d'affirmer sa différence face à l'Etat ou de générer des soutiens pour la mise en œuvre locale des politiques publiques. D'un autre côté cependant, la mobilisation de la part des populations d'appartenances multiples et la prolifération des références identitaires - phénomène qui dépasse de loin le seul exemple des Antilles françaises - contraint fortement la formulation de ces affirmations et rend problématique la recherche, fortement asservie aux logiques de conquête de nouveaux espaces de pouvoir, d'une hypothétique coïncidence entre identité politique et identité culturelle qui anime les mouvements nationalistes. 


\section{Subordination coloniale et recomposition identitaire}

Dans toute société, l'acteur social se trouve à l'intersection de plusieurs groupes d'appartenance et peut changer ses affiliations et ses degrés d'obédience. La fonction des discours identitaires est alors d'orienter ce choix et de créer les conditions d'une adhésion privilégiée à un groupe particulier [Martin, 1994 : 23], notamment dans les situations potentiellement riches en conflits d'allégeances, comme c'est le cas pour les expériences coloniales. Deux séries de phénomènes ont pourtant contribué à relativiser, voire à masquer la perception de telles attitudes aux Antilles françaises: d'une part, la volonté longtemps affichée par le pouvoir central français d'éradiquer les lieux de résistance collective à travers les tentatives répétées d'assignation d'une identité par le haut ainsi que l'alignement sur les institutions politiques de la métropole ; d'autre part, et corrélativement la prégnance, jusqu'à l'orée des années 80, d'un discours savant, fréquemment articulé au combat militant, fondé sur la rhétorique de la dénonciation de l'aliénation coloniale. Ces deux postures - qui tendent à se conforter mutuellement - ont longtemps entretenu l'illusion d'une stricte assimilation aux normes et valeurs métropolitaines, voire d'une totale acculturation. Pourtant, la réalité se révèle bien plus subtile et complexe : elle dévoile la formation et la perpétuation de comportements sociaux et de sphères d'activités culturelles échappant largement aux normes imposées. Et s'il est vrai que les clivages nés de la subordination coloniale ont durablement influé sur la formation des 
identités aux Antilles françaises, le processus est loin d'être univoque : les tentatives d'assignation identitaire suscitent en retour des stratégies défensives, mais aussi d'adaptation, voire d'instrumentalisation. Exemplaire est à cet égard la façon dont le mythe républicain d'égalité a été investi localement.

Différents travaux ont depuis longtemps souligné quelques uns des paradoxes majeurs de l'histoire coloniale des Antilles. Sous-tendu par un idéal assimilationniste dont les traces profondes sont perceptibles dès l'Ancien Régime, nourri d'une prétention universaliste que l'héritage révolutionnaire n'a cessé de conforter, le projet colonial qui s'y déploie n'a pas moins été une «formidable machine à produire de la différence » [Giraud, 1997]. Le rapprochement de peuples d'horizons extrêmement divers débouchant sur la formation de sociétés - selon une trajectoire historique dont on ne soulignera jamais assez la singularité - a été un puissant facteur de création d'espaces culturels et d'espaces sociaux qui ont tenu en respect la dynamique assimilatrice. S'il est donc incontestable que le dispositif colonial et étatique français, particulièrement après la départementalisation, a longtemps été réfractaire à une quelconque forme d'autonomie culturelle et politique, il n'en demeure pas moins qu'il n’a jamais débouché, contrairement à une idée largement répandue, sur un strict alignement sur les normes métropolitaines.

En outre, les processus de confinement et de marginalisation des groupes dominés dans des cadres délibérément inégalitaires n'ont pas peu contribué à l'émergence d'identités propres à ces groupes. Pour ces derniers, la conquête de l'égalité sociale 
inhérente à la citoyenneté ne pouvait passer que par la revendication de particularités culturelles [Suvélor, 1983; Giraud, op. cit.]. En effet, le caractère profondément inégalitaire, hiérarchisé et compartimenté des sociétés insulaires a conduit les anciens esclaves nouvellement libérés à rechercher le salut en direction de la métropole républicaine dès la deuxième moitié du XIXème siècle. Située dans le prolongement de ces aspirations, l'assimilation institutionnelle réclamée par Aimé Césaire en 1946 vise, sous un certain rapport, à réconcilier la revendication de l'égalité et celle de la spécificité, ou plus exactement à permettre l'accès à l'Universel par la lutte contre les inégalités et la réaffirmation des valeurs noires jusqu'alors bafouées. Toutefois, elle est bientôt désavouée par son propre promoteur parce que trop peu respectueuse des particularismes culturels, sans permettre pour autant de répondre aux attentes pressantes en matière d'égalité sociale.

Les tentatives de nationalisation de la culture locale, la condamnation sans appel de l'un de ses vecteurs structurants, la langue créole, et la relégation de cette culture au rang de pratiques folklorisées au nom de l'idéal républicain d'émancipation n'ont pas été simplement imposées par le Centre. En réalité, elles ont bénéficié du soutien de certaines catégories sociales locales et ont parfois correspondu à des dynamiques et à des demandes émanant des sociétés insulaires, au point d'accréditer l'idée d'une « assimilation pure et parfaite ». Pour autant, il serait absurde de prétendre que la culture nationale française s'est entièrement substituée à la culture périphérique. Les phénomènes de contact, y compris dans les situations d'oppression et d'inégalité, ont 
des effets plus délicats à analyser que la simple imitation ou assimilation des traits d'un groupe par un autre dans la mesure où ils provoquent des échanges réciproques susceptibles de modifier les attitudes des uns et des autres [Jules-Rosette, Martin : 1997]. Nul doute, de ce point de vue, que l'expérience identitaire aux Antilles françaises se caractérise par un ré-agencement fondé sur une superposition d'appartenances subjectives. Sans conteste, la force assimilatrice de l'État a très largement résidé dans son indéniable capacité à tolérer un espace insulaire médiatisant l'appartenance à une communauté élargie. Cet espace insulaire a été le lieu où s'est opérée la réactivation du sentiment d'appartenance à la société locale.

Un tel mécanisme a été assuré, dans le cadre du système politico-administratif mis en place avec la départementalisation, par les représentants des «communautés » insulaires qui en accédant aux ressources étatiques dans une quête effrénée d'égalité ont décliné, sur un mode mineur le plus souvent, les particularismes compatibles avec le maintien au sein de l'ensemble national français. Dans leur fonctionnement quotidien et dans leurs rapports avec les fonctionnaires délégués par le centre, ils ont contribué à une certaine autonomisation des espaces politiques insulaires [Daniel, 1997]. Ce système a favorisé, face aux déconvenues et désillusions engendrées par l'échec de la départementalisation, un revivalisme des formes culturelles autochtones. En effet, "la survalorisation sociale de la culture "classique" française et sa contrepartie, la minoration des cultures antillaises, étaient intrinsèquement liées à la crédibilité de l'idéologie assimilationniste dont ladite départementalisation a été le produit 
majeur. Une fois cette crédibilité atteinte à travers les contradictions et les contrariétés du statut départemental, les situations culturelles antillaises ne pouvaient donc pas manquer d'en être affectées» [Giraud, 1997, op. cit. : 385]. En d'autres termes, le mythe de l'égalité républicaine, tout en continuant de parcourir l'imaginaire collectif et de servir de fondement à la légitimité élective des élus, s'est progressivement affaibli, ouvrant la voie à une réactivation des demandes spécifiques adressées au centre et à une politisation des identités antillaises.

\section{- Le passage au politique de la revendication identitaire}

La politisation des identités obéit à des logiques multiples et variées. Elle est le plus souvent symptomatique d'une crise du rapport à l'État. Dans le cas des Antilles françaises, il s'agit plus précisément d'une contestation du modèle traditionnel d'intégration politique. Nourrie des contrariétés du statut départemental, cette crise favorise la territorialisation des structures partisanes ainsi qu'une forte valorisation $\mathrm{du} \ll$ local $»$

- La territorialisation du politique

Le processus a puisé, dans un premier temps, son élan dans les conflits qui se sont noués autour de l'expérience de la départementalisation avant de s'intensifier et de s'étendre à l'ensemble des forces politiques, sous la forme d'une distanciation croissante à l'égard des appareils partisans métropolitains. 
Appliqué aux Antilles françaises, le jacobinisme bien connu de l'administration française suscite, dès la fin des années 50 , des revendications en faveur du respect des spécificités et d'un renforcement de l'autonomie interne au terme d'un renversement de situation : les thèmes de l'autonomie et des «spécificités » que la droite s'était jadis appropriés sont alors investis par les partis de gauche ${ }^{1}$. Dès lors, la vie politique cristallise, sur un mode quelque peu schématique, une opposition entre les trois adversaires principaux du jeu politique : les tenants d'une assimilation politique et culturelle et donc d'une identité recomposée par l'État français, les protagonistes d'une autonomie culturelle aménagée au sein de l'ensemble français et respectueuse de la différence et, enfin, les partisans d'une altérité radicale. La première attitude articule très clairement un thème électoralement porteur, l'accession à tous les droits-créances inhérents à la citoyenneté française, à une valorisation de la culture métropolitaine. La deuxième vise principalement à concilier deux démarches : d'un côté l'énonciation d'un discours centré sur des thèmes, d'une efficacité électorale moindre, tels que le respect de l'identité culturelle, la nécessité de repenser le modèle de développement et le renforcement des pouvoirs locaux ; de l'autre, l'acceptation d'une logique de dépendance présidant au fonctionnement des institutions départementales et assortie de l'application des droits sociaux. La troisième plaide en faveur de l'accession à la souveraineté et réalise des scores confidentiels au plan électoral.

1. Il s'agit du Parti Progressiste Martiniquais, du Parti Communiste Martiniquais et du Parti Communiste Guadeloupéen. 
L'essoufflement du mythe républicain, associé à partir des années 80 à la montée des incertitudes liées à la construction européenne, contribue à la redéfinition de ces stratégies politiques et identitaires. Il libère, en effet, un fort potentiel contestataire et favorise la multiplication des proclamations identitaires - dont la traduction électorale demeure néanmoins problématique - tout au long des années 70. Le phénomène s'accélère néanmoins à la faveur de la décentralisation intervenue au début des années 80 . Cette dernière se traduit par une relative dépolarisation dans les relations entre le centre et la périphérie, un désenclavement des forces autonomistes portées à la tête des institutions décentralisées et la montée en puissance des mouvements nationalistes. L'UPLG ${ }^{2}$ en Guadeloupe et surtout le $\mathrm{MIM}^{3}$ à la Martinique - capitalisent lors d'élections successives les craintes suscitées par l'absorption dans l'ensemble européen en développant un discours, partiellement relayé par les forces autonomistes et popularisé par une célèbre chanson de carnaval ${ }^{4}$, sur les risques d'une dilution des différences culturelles. Ils cristallisent également un mécontentement multiforme en tirant profit d'une certaine désaffection à l'égard de la gestion des institutions décentralisées et de l'incapacité des partis politiques traditionnels à construire un discours réellement novateur répondant aux attentes des populations. Paradoxalement cependant, leur audience s'accroît à mesure que les perspectives d'accession à la souveraineté semblent

\footnotetext{
2. L'Union Pour la Libération de la Guadeloupe.

3 . Mouvement Indépendantiste Martiniquais.

${ }^{4}$. La signature de l'Acte unique européen a donné lieu à une chanson populaire ayant connu un vif succès mettant en scène un agneau - les Antilles et la Guyane - livré à l'appétit féroce d'un loup, l'Europe (voir annexe).
} 
s'éloigner. Les alternances politiques successives intervenues en France depuis 1981 qui tendent à discréditer «le chantage au largage» longtemps pratiqué par la mouvance départementaliste, la gestion des institutions décentralisées par les forces autonomistes et, corrélativement, la dépolarisation sus-mentionnée entre le centre et la périphérie, le retrait de l'Etat qui abdique une partie de son ambition dans le domaine culturel sont autant de facteurs susceptibles d'expliquer ce paradoxe apparent. Ils concourent, dans une large mesure, à banaliser la revendication indépendantiste à la Martinique - le MIM gère la région depuis 1998 avec le soutien de la droite locale -, à l'affaiblir en Guadeloupe, et surtout à libérer un espace d'expressions identitaires et d'affirmation des particularismes compatible avec la double appartenance, unanimement revendiquée, aux ensembles français et européen.

Il convient d'ajouter que cette évolution n'épargne pas les formations et les responsables politiques d'une frange de la droite classique. Celle-ci cesse de remettre en cause l'existence des cultures locales et défend désormais les identités insulaires. En outre elle amorce, une trentaine d'années après les partis de gauche, une stratégie de territorialisation, perceptible dès la fin des années 80 , à travers le lancement, resté sans lendemain, de Martinique avant tout du leader gaulliste, mais aussi départementaliste intransigeant, Michel Renard. Plus récemment, le mouvement Osons oser créé par le député Pierre Petit, lui aussi issu de la mouvance gaulliste, tente d'associer, à l'instar d'Objectif Guadeloupe mis en place dans l'île sœur par 
Lucette Michaux-Chevry, le respect des droits inhérents à la citoyenneté française, la défense des racines locales et le renforcement du pouvoir local.

Cette distanciation de certains partis ou mouvements politiques incarnant la droite traditionnelle à l'égard des appareils partisans métropolitains comporte une double conséquence. D'une part, combinée à l'affaiblissement généralisé des structures partisanes, elle contribue à accroître la fragmentation de paysages politiques qui semblent évoluer par scissiparité. On assiste en effet à la multiplication de groupements politiques dont certains n'ont qu'un simple fondement mayoral. Phénomène qui se renforce d'une actualisation des stratégies de conquête des postes électifs de plus en plus tributaires de la mobilisation de ressources exclusivement locales, l'alliance avec les forces politiques nationales, naguère privilégiée et recherchée, n'étant plus considérée comme un atout suffisant. D'où le repli de plus en plus manifeste sur des micro-identités, conduisant parfois à mettre le local à distance, non seulement des enjeux centraux et globaux, mais des débats censés structurer, à l'échelle des pays, les choix partisans. Paradoxe supplémentaire : ces clôtures identitaires se mettent en place précisément à un moment où se développe une véritable mythologie quant à la nécessité d'une approche globale des problèmes. Ainsi, la dernière campagne des élections cantonales et municipales a-t-elle été largement déconnectée du débat sur l'avenir institutionnel, lequel pourtant a largement meublé, sur un mode souvent passionné, la vie politique tout au long de la période précédente. D'autre part, l'ébauche de territorialisation des mouvements 
politiques de droite va de pair avec une mutation de leur registre discursif. Leurs leaders tentent de puiser leur légitimité non plus uniquement dans des alliances avec les partis métropolitains, mais dans la référence à une communauté spécifique, historiquement constituée et dont l'homogénéité culturelle est en permanence rappelée. Pour ce faire, ils n'hésitent pas, dans un registre qui ne déplaît pas à la mouvance indépendantiste et qui tend à les rapprocher des «rassembleurs » entretenant une proximité certaine avec le populisme [Hermet, 2001: 429] à «flatter les préjugés populaires et à sceller l'alliance des patriotes de toutes provenances afin de sauvegarder le pays authentique » [Hermet, op. cit.].

Ce n'est pas dire pour autant que les élites politiques, indépendamment de leurs options idéologiques, négligent le recours aux ressources centrales. Mieux: les spécificités locales valorisées par le débat politique s'accommodent fort bien de la référence alternative à un système de normes centrales et universelles. En d'autres termes, dopées par l'épuisement du discours fondé sur l'égalitarisme républicain, les proclamations identitaires et les revendications statutaires ne sont nullement exclusives de la recherche simultanée d'alliés au cœur des institutions centrales. Ainsi la Présidente de la collectivité régionale guadeloupéenne fait-elle constamment valoir ses relations privilégiées avec le Président Chirac afin de construire son leadership en matière de coopération régionale; de même, le Président du Conseil général de la Martinique a-t-il profité de ses rapports étroits avec le Gouvernement de Lionel Jospin pour conforter sa stature de leader politique. Dans tous le cas 
cependant, ces attitudes vont de pair avec la dénonciation, modulée en fonction de la nature du Pouvoir à Paris, d'un Etat jugé peu respectueux des particularismes locaux et des identités insulaires. Rapportées aux intérêts politiques qui guident l'action des acteurs, elles rappellent le caractère dynamique des identités qui ne sont jamais que le produit contingent et évolutif des luttes entre acteurs sociaux qui s'affrontent pour sa définition et/ou pour le pouvoir, des rapports de force entre groupes ainsi que des mobilisations collectives générées. Construction évolutive et dynamique, les identités servent de support à des stratégies nécessairement finalisées lesquelles « apparaissent comme le résultat de l'élaboration individuelle et collective des acteurs et expriment, dans leur mouvance, les ajustements opérés, au jour le jour, en fonction des situations et des enjeux qu'elles suscitent-c'est-à-dire des finalités exprimées par les acteurs - et des ressources de ceux-ci » [Taboa Leonetti, $1990: 49]$.

Ainsi la distanciation des partis politiques locaux à l'égard des appareils partisans métropolitains, remontant aux années 50 pour la Gauche, plus récente pour la droite n'obéit pas nécessairement à une tendance linéaire et univoque. Elle reste fortement tributaire des logiques de conquête des postes électifs et s'ajuste en fonction des enjeux du moment et des ressources mobilisables. Favorisée par l'exacerbation des affirmations identitaires au cours de ces dernières années, elle s'accompagne et se renforce d'une forte valorisation du local perceptible dans de multiples champs sociaux que le politique n’hésite plus à investir.

- La valorisation des ressources politiques locales 
Deux exemples, parmi beaucoup d'autres, témoignent de cette dynamique de valorisation $\mathrm{du}$ local. A commencer tout d'abord par une véritable explosion d'activités dans le domaine culturel. Chasse gardée pendant longtemps des mouvements autonomistes et indépendantistes, les cultures locales font désormais l'objet d'un intense investissement dans la quasi-totalité des secteurs de l'opinion. Il est vrai que le pouvoir central, longtemps réfractaire à toute forme d'expression publique des identités périphériques, admet désormais l'existence de cultures à partir de laquelle s'énonce la différence au point de participer financièrement à leur valorisation. A la faveur du relâchement des tensions entre pouvoir central et pouvoirs locaux, les collectivités territoriales multiplient de leur côté, de manière souvent désordonnée et selon une démarche qui privilégie la logique des équipements collectifs au détriment d'objectifs clairement définis, les initiatives et les actions dans le domaine culturel [Bernabé, Capgras, Mugier, 1997]. Ce nouvel engouement pour la «chose culturelle » est lourd d'ambiguïtés et de paradoxes : il s'accompagne, de la part de des responsables politiques le plus souvent imprégnés d'une culture de contestation du pouvoir central, de l'appel à l'expertise nationale, de la quête d'une sorte d'adoubement synonyme de reconnaissance nationale [Constant, 1993]. L'objectif est pourtant clair : il s'agit de damer le pion à un Etat délibérément en retrait - au risque d'alimenter une sourde compétition entre collectivités ainsi que les porteurs de projets tentés, chacun en ce qui le concerne, de mettre en avant ses propres particularismes - afin de circonscrire des espaces symboliques de réhabilitation des cultures locales et d'affirmation de soi. 
En second lieu, et dans un registre plus général, les élus procèdent, dans leurs rapports avec les centres métropolitain et européen, à une mise en scène permanente des «spécificités » érigées en véritables emblèmes d'une identité ainsi recomposée. Nul doute, en effet, que le terme «spécificité » n'a jamais connu pareille fortune, tant il est fréquemment mobilisé par le personnel politique local, toutes tendances politiques confondues, dans le cadre des négociations avec les autorités centrales et/ou européennes. Autrement dit, les proclamations identitaires sont de plus en plus instrumentalisées par les collectivités territoriales en vue de générer des soutiens aux politiques publiques localement mises en œuvre. La lutte pour la maîtrise du territoire et pour l'hégémonie dans le cadre du partenariat avec l'État et l’Union européenne ainsi que la constitution du leadership local s'appuient très largement sur le recours aux notions de «dignité» et de «spécificités» qui participent de la construction symbolique d'identités collectives. Désormais, un leader politique ne tire plus, comme par le passé, sa force de son habileté à monopoliser la médiation entre les populations et le pouvoir central. Sa capacité à s'ériger en entrepreneur identitaire, apte à valoriser les ressources politiques locales et à faire vibrer la fibre identitaire à travers les échanges politico-symboliques compte tout autant que le label de bon gestionnaire ou celui de médiateur. Les rapports plus ou moins conflictuels entre les exécutifs départementaux et/ou régionaux et les préfets ne sont pas la simple résultante d'un découpage de compétences, à bien des égards, irrationnel et source de complexités. Ils s'expliquent tout autant par la volonté de s'imposer symboliquement face à l'Etat, en le délégitimant dans sa capacité à 
élaborer et mettre en œuvre des politiques publiques adaptées aux contextes locaux, grâce à la manipulation d'un répertoire faisant sens au regard des réalités socioculturelles. De même, la dénonciation récurrente de "l'incapacité à gérer un pays éloigné comprenant un peuple de culture distincte et différente, à partir d'un centre hors de nos réalités et de nos sensibilités » [Manifeste, 2000] ${ }^{5}$ constitue un véritable plaidoyer en faveur d'un « retour au local » assorti d'une forte connotation identitaire. Significatif est à cet égard le débat qui s'est noué autour de deux textes, le projet de loi d'orientation élaboré par le gouvernement après une longue procédure de consultation et la Déclaration de Basse-Terre signée par les Présidents des conseils régionaux de la Guadeloupe, de la Guyane et de la Martinique. Il s'agit très clairement, dans le second cas, de prendre le contre-pied d'une démarche procédant d'en haut: le local est érigé en réceptacle des identités particularistes et en authentique espace d'émancipation, seul capable de stabiliser la revendication identitaire. La volonté affichée, au moins d'un point de vue politico-symbolique par l'ensemble du personnel politique de produire des projets globaux - guadeloupéen et martiniquais - obéit à la même logique. Alimentée par le mythe de la "proximité » qui associe dans une relation mécanique renforcement des pouvoirs locaux, démocratie, et développement, elle entend mettre à distance l'Etat en privilégiant une démarche qui tend à rejeter tout principe supérieur unificateur au profit des particularismes adossés à des considérations d'ordre culturel et considérés comme chargés de vertu et non négociables.

5. Il s'agit d'un manifeste en faveur de l'autonomie signé par 27 dirigeants et membres du PPM. 
Pourtant, de même que la territorialisation des forces politiques n'est nullement exclusive de stratégies d'alliances, largement contextualisées, avec les partis métropolitains, la promotion d'un local à forte connotation identitaire s'avère pleinement compatible avec la mobilisation de ressources - essentiellement matérielles cette fois - aux niveaux central et européen. Il n'existe pas en effet de correspondance parfaite entre l'homogénéité culturelle postulée et l'action proprement politique. Ainsi, après les craintes suscitées au moment de la signature de l'Acte unique, l'Europe semble avoir en quelques années troqué dans l'imaginaire de certains, en particulier les élus, "le statut de loup contre celui, non moins fantasmatique, de véritable vache à lait » [Lise, 2001]. De telles attitudes que l'on pourrait tout aussi aisément repérer dans les rapports avec l'Etat révèlent en réalité la fluidité des allégeances ainsi que la forte porosité des clôtures identitaires. Simultanément insérés dans une pluralité d'espaces économiques, sociaux et culturels, guidés par différents niveaux et principes d'identification, les acteurs sociaux sont porteurs d'identités mobiles et changeantes. C'est la raison pour laquelle, à distance de toute conception d'une identité «naturelle» s'imposant aux individus et aux groupes comme un attribut immuable, les expériences antillaises invitent à réfléchir aux stratégies identitaires rationnellement conduites par des acteurs eux mêmes identifiables [Bayart : 1996].

Au total, la politisation des identités apparaît comme un élément stratégique et une importante ressource idéologique pour les groupes engagés dans une lutte pour le 
pouvoir s'organisant autour de programmes économiques, politiques et sociaux plus ou moins conditionnés par la question du statut. En particulier, chaque mouvement politique travaille à la construction d'une identité culturelle dont la coïncidence, fréquemment postulée, avec une identité politique également construite demeure pour le moins problématique.

- Les contrariétés de la «souveraineté identitaire $»^{6}$

De toute évidence, les mouvements nationalistes semblent avoir renoncé, au moins provisoirement, à leur prétention en matière de souveraineté politique. Plus exactement, ils semblent l'avoir troquée contre une forme de "souveraineté identitaire » qui s'actualise au plan politique dans une revendication pour le moins euphémisée: le renforcement du pouvoir local. Nourries par la montée des affirmations identitaires, les propositions d'évolution institutionnelle sont néanmoins infléchies et parasitées par des considérations tactiques et stratégiques directement liées au fonctionnement des assemblées départementales et régionales et aux processus de construction de leadership en leur sein [Daniel, 2000a : 89]. Tel est, par exemple, le sens de «l'alliance de gestion » mise en place à la Martinique au lendemain des élections régionales de mars 1998 et qui a conduit la droite locale - dont une frange pratique volontiers le «transformisme » politique en captant les thèmes de ses adversaires d'hier - à gérer l'institution régionale aux côtés du MIM. En Guadeloupe, l'éclatement du paysage politique associé aux divisions de la gauche classique a

\footnotetext{
${ }^{6}$. Nous empruntons cette expression à Michel Graud [Giraud, 2000, op.cit]
} 
permis à Lucette Michaux-Chevry, Présidente de la région et leader charismatique de la droite locale, d'agréger autour d'elle une clientèle d'élus venant d'horizons divers. Désormais, elle incarne la revendication d'autonomie à laquelle s'est rallié le mouvement nationaliste manifestement en perte de vitesse sur le plan électoral et, pour tout dire, cherche à capitaliser l'exacerbation d'une forme de conscience identitaire. Dotés parfois d'une faible lisibilité pour les citoyens dans la mesure où ils brouillent les domaines classiquement réservés à la droite et à la gauche et/ou circonscrits par le traditionnel débat statutaire, les choix ainsi opérés, sur la base d'une réduction culturaliste et homogénéisatrice du politique, reposent sur un ensemble de postulats qu'il convient d'objectiver en même temps qu'ils sont révélateurs d'une série de paradoxes, voire d'apories qui rendent d'autant plus complexe la gestion d'un phénomène têtu d'allégeances multiples. De ce point de vue, trois séries de remarques s'imposent.

En premier lieu, la réduction culturaliste du politique conduit à sous-estimer la pluralité des affiliations identitaires qui tend à s'affirmer au sein des sociétés antillaises. Combinée à un affaiblissement de la capacité des institutions - syndicats et partis politiques notamment - à canaliser et à articuler les demandes sociales, cette situation se traduit pourtant par une diversification des intérêts et une nette tendance des acteurs sociaux à reconstruire leur subjectivité en termes d'identité culturelle, comme le révèle l'analyse des conflits sociaux à la Martinique [Daniel, 2000b]. D'où, 
par exemple, la prolifération de single issue groups ${ }^{7}$ qui surgissent sur la scène sociale et politique, défiant parfois les canaux traditionnels d'expression et valorisant leurs particularismes sociaux et culturels considérés d'emblée comme étant chargés de vertu. En affirmant de manière vigoureuse leur singularité, de tels groupes accréditent l'idée qu'aucune instance de représentation ou de médiation - élus, partis, institutions et mouvements politiques... - n'est capable, à elle seule, de réaliser la plénitude d'une communauté dont l'homogénéité culturelle est soumise à rude épreuve. Procédant parfois d'une sorte $\mathrm{d}^{\prime}$ «essentialisme stratégique », mobilisant de manière simultanée ou successive des répertoires variés - affectifs mais aussi utilitaristes, voire manipulatoires - en fonction des contextes, ils énoncent des stratégies qui rappellent une banalité, lourde de sens dans le cas des sociétés antillaises : 1 'identité est par définition plurielle et composite.

En second lieu, le travers culturaliste va de pair avec une représentation moniste du politique qui postule l'existence, sous une forme idéalisée, du peuple comme totalité homogène, transparente à elle même et incarnant une «souveraineté » immédiatement lisible et opérationnelle. Ainsi, l'un des prolongements de la Déclaration de Basse-Terre a consisté à adresser à l'ensemble de la population un questionnaire censé donner la parole aux Guadeloupéens sur l'avenir qu'il souhaite pour leur pays. On ne s'arrêtera guère sur les problèmes posés par le questionnaire lui même, en particulier la formulation biaisée de certaines questions ni sur l'impossibilité de contrôler la

\footnotetext{
7. Groupes à vocation exclusive, défendant un intérêt unique, sectoriel et cloisonné, et fondant parfois leur revendication sur des particularismes présentés comme non négociables.
} 
validité des réponses et leur représentativité - une même personne pouvant expédier plusieurs formulaires - pour s'en tenir à une rapide appréciation sur la signification d'une telle démarche. Celle-ci revient à considérer que le peuple «existe comme un donné immédiatement cohérent, à l'identité évidente, suffisamment déterminée par la confrontation à un "extérieur" » [Rosanvallon, 2000 : 418]. Paraphrasant le titre d'un célèbre article de Pierre Bourdieu [Bourdieu, 1973], on pourrait au contraire légitimement prétendre que le «peuple n'existe pas»sous la forme d'un acteur homogène et continûment actif, pré-existant à l'activité politique et porteur d'un projet fini, mais à travers un processus permanent d'interaction avec ses représentants et la libre confrontation, au sein de l'espace public, de points de vue particuliers. Il est vrai cependant que le questionnaire sus-mentionné n’a pas pour ambition première de recueillir une «opinion» dont les rares indices disponibles tendent à montrer qu'elle est loin d'être constituée, a fortiori sur un mode unanime. Elle vise plutôt à légitimer une démarche célébrant l'existence d'une communauté politique solidaire, guérie de ses désunions artificielles et capable d'un sursaut promothéen, dans la perspective d'une re-fondation de l'avenir du pays et dans un rapport d'opposition à un projet gouvernemental - la loi d'orientation pour l'outre mer - frappé d'une altérité radicale. Si l'approche a été quelque peu différente à la Martinique, elle ne pose pas moins des problèmes analogues. Ici le «consensus martiniquais » est exalté à travers une démarche qui procède à la valorisation de la société civile et qui prétend évacuer toute conflictualité dans des forums ou ateliers 
ouverts à l'ensemble de la population ${ }^{8}$. Parée de toute les vertus, la société civile en arrive à incarner, le mythe de la démocratie participative aidant, un projet cohérent et mobilisateur, conçu avec les élus, les «experts » et les «personnes ressources ». Il est clair, pourtant, qu'une telle approche, si généreuse soit-elle dans son principe, néglige les effets de «cens ${ }^{9}$ social » et les inégalités qui sont à l'œuvre dans les espaces publics ainsi forgés. Elle ne tient nullement compte d'un constat, somme toute, banal : la participation se limite aux personnes déjà intéressées et motivées et qui, le plus souvent, représentent des groupes en lutte pour leur reconnaissance ou le pouvoir et producteurs d'identités particulières [Neveu, 1999]. D'où le contraste frappant entre l'intense mobilisation des élus et mouvements politiques autour de la question statutaire censée structurée une identité politique forte d'un côté, et de l'autre l'apparente faiblesse d'une citoyenneté tentée par l'indifférence ou entièrement asservie à une logique instrumentale de simple exercice de droits.

Enfin, l'évolution récente se caractérise certes par un recentrage des mouvements nationalistes ou porteurs d'affirmations identitaires. Mais ce recentrage n'obéit pas simplement à des exigences d'ordre tactique; il reflète surtout une incapacité à produire un discours alternatif capable de se substituer au mythe républicain et un projet politique susceptible de supplanter l'allégeance citoyenne ainsi que l'identité

\footnotetext{
8. "L'essentiel est que les Martiniquais que nous avons côtoyés pendant ces deux mois, appartenant à des milieux socioprofessionnels très différents, ayant des positions idéologiques et des appartenances politiques dont on pensait jusqu'à présent qu'elles étaient inconciliables ont, comme le Président Alfred Marie-Jeanne nous le disait le 06 octobre "laissé leurs contradictions au parking" » [Projet Martinique, 2000 : 3]

9. L'expression " cens » rappelle ici le "système censitaire », qui subordonnait la participation politique au paiement d'un minimum d'impôt, le cens, et qui excluait par là-même une majorité de citoyens.
} 
politique officiellement proclamée par l’Etat [Dahomay, 2000 ; Trésor, 1999]. Sans doute ces différents mouvements ont-ils été, on le répète, à l'origine d'un renouvellement de la question de l'identité culturelle au cours des trois dernières décennies : ils ont largement occupé le champ culturel, soit, de manière générale, en forgeant un espace d'autonomie et de refuge face aux velléités totalisantes de l'Etat, soit par l'intermédiaire d'écrivains de renom dont certains ont été tentés de passer au politique, soit, enfin, par des opérations de valorisation du patrimoine (création d'éco-musées, organisation de festivals culturels, défense de la langue créole...).

\section{- Le passage au politique des intellectuels}

Ces dernières années ont été marquées par un intense débat sur l'avenir institutionnel et le développement des Antilles françaises et de la Guyane. En témoigne cet extrait du Manifeste pour refonder les DOM signé par quatre intellectuels qui, après avoir fait l'objet d'une large diffusion, notamment sur internet, a été publié par le Monde du 21 janvier 2000.

\section{Convenir d'un projet qui nous rassemble est un acte fondateur}

Que pouvons-nous faire ensemble pour exister au monde ? Comment concilier notre nécessaire responsabilité collective avec les réalités économiques universellement et férocement triomphantes? Le moment est venu de débattre publiquement de ce que beaucoup d'entre nous pensent, l'un à part l'autre.

La transformation progressive du tissu économique et social de la Guadeloupe, de la Guyane, de la Martinique ne peut se faire en dehors de la mise en oeuvre d'un projet global qui préparerait l'avenir en tenant compte du présent.

Nos pays ne peuvent envisager de solutions à mettre en oeuvre, ni leur calendrier, dans la perspective, par exemple, d'une industrialisation lourde, d'une agriculture extensive, d'un tourisme exclusif ou d'une hypertrophie du secteur tertiaire, commerce de consommation, etc... Dans tous ces domaines, notre retard ou nos impossibles sont irrattrapables.

Notre seule ouverture est celle d'une production diversifiée à valeur ajoutée, (la valeur ajoutée étant cette donnée spécifique dont l'exploitation permet un profit et un développement réel) comme celles qu'ont mises en oeuvre la plupart des petits pays 
qui aujourd'hui disposent de leur propre sort. Dans le concert baroque du monde actuel, nous croyons à l'avenir des petits pays. Autrement dit, nous croyons qu'il n'y a pas de petits pays, il n'y a que de grands projets.

Les conditions générales des Antilles, de la Guyane et de la Caraïbe (des îles, ou des espaces facilement nettoyables, aisément transformables) font que la valeur ajoutée que nous pouvons envisager résulterait d'une production à caractère biologique, dont la demande grandit irrésistiblement sur le marché mondial. Il nous faut occuper ce créneau.

C'est pourquoi, depuis quelques temps déjà, certains d'entre nous ont proposé de mettre en place en Martinique le projet global d'une économie centrée sur des produits biologiques diversifiés, et de conquérir sur le marché mondial le label irréfutable «Martinique pays à production biologique», ou «Martinique, premier pays biologique du monde ».

Nous appelons les Guadeloupéens, les Guyanais et les Martiniquais à considérer la nécessité d'une telle orientation, même si dans chacun de ces pays un projet de cette nature peut passer par des voies différentes, par exemple un accomplissement technologique en Guyane.

En ce qui concerne le projet biologique les difficultés sont immenses et nous n'en signalons ici que quelques-unes de caractère très général.

Un tel projet ne saurait être imposé d'en haut, il devrait être l'affaire de tous, débattu par tous. Il ne serait pas viable si une seule composante de notre réalité s'en tenait à l'écart ou en était exclue, par quelque mécanisme ou quelque préjugé que ce soit.

Il ne serait pas viable sans l'adhésion de la jeunesse, qui y trouvera des motifs d'enthousiasme et d'action. L'insertion et l'emploi sont irréalisables en dehors d'une intention collective et d'une visée commune.

Il ne serait pas viable s'il ne favorisait pas, dans la chair même de notre lieu, des vitalités culturelles, linguistiques et artistiques, capables d'éveiller notre regard et de renouveler notre imaginaire de nous-mêmes et du monde. Ce projet ne serait pas viable s'il n'est pas total, c'est à dire s'il n'englobe pas tous les secteurs d'activité, agriculture, tourisme, agroalimentaire, médecine, pêche, communication, lutte contre la pollution, système éducatif, secteurs de production et de consommation, etc. Toute entreprise biologique isolée est fragile et périssable.

Patrick CHAMOISEAU (Martinique) est écrivain

Gérard DELVER (Guadeloupe) est directeur culturel de la ville de Basse-Terre

Edouard GLISSANT (Martinique) est écrivain

Bertène JUMINER (Guyane) est écrivain, professeur des universités 
Mais en faisant de la reconnaissance de la culture le champ privilégié de leur action en dépit des tentatives de renforcement des forces sociales à travers les syndicats qui ont certes connu un processus de territorialisation identique à celui inauguré plus tôt par les structures partisanes -, ils ont négligé la complexité des rapports entre identité culturelle et identité politique. Tentant vainement de rabattre l'une sur l'autre, le discours indépendantiste a fini par s'épuiser dans l'incantatoire en se diluant dans un contexte propice à la mobilisation d'appartenances multiples de la part des populations. C'est peu dire, en effet, que la départementalisation « a modifié les processus d'identité en empêchant qu'ils se rabattent sur un substrat ethnique, en les rendant réflexives, et en permettant, pour parler comme Habermas, que se développe "un contexte intersubjectivement partagé d'entente possible" » de nature à fortifier le désir d'intégration républicaine [Dahomay, op. cit. : 115]. Et ce n'est pas le moindre des paradoxes que de constater que l'abandon - provisoire ? - de la stratégie de rupture est loin d'être la conséquence d'une offensive politique organisée des partisans de la départementalisation: orphelins depuis une vingtaine d'années de leur propre stratégie de conquête des postes électifs, inaptes à renouveler leur discours, ces derniers sont désormais figés dans une posture strictement défensive peu compatible avec l'élaboration d'un projet politique structuré. En réalité, la difficulté pour les mouvements nationalistes ou identitaires à s'imposer résulte d'un double constat convergent : leur incapacité à apporter, dans le cadre d'un projet en cohérence avec l'idéal indépendantiste ou autonomiste « des réponses crédibles aux interrogations diverses et légitimes d'une population sans doute sensible au fait identitaire, mais qu'une 
réalité complexe oblige à beaucoup de discernement dans le choix de ses options politiques » [Trésor, op.cit: 30]; la tentation récurrente de procéder à une superposition de l'identité politique et de l'identité culturelle. Sur le premier point, force est de reconnaître que même le mouvement de la créolité qui prétend défendre, dans le champ littéraire et artistique, une «identité mosaïque », à distance de toute conception essentialiste tout en prétendant lui conférer un prolongement sur le plan politique [Bernabe et alii, 1989] semble avoir échoué à contourner le dilemme des allégeances multiples: il n'a pas réussi à ce jour à proposer un projet politique clairement articulé, à la hauteur en tout cas de l'ambition affichée. Et ce n'est guère le Manifeste pour un projet global signé par l'un des inspirateurs du mouvement, Edouard Glissant, et l'un de ses fondateurs, Patrick Chamoiseau ${ }^{10}$, qui semble de nature à combler ce vide relatif. S'il tente de dépasser l'affirmation rituelle sur la nécessité d'un projet, en tenant pour principale cette dernière et secondaire la question du statut, c'est pour, à côté de l'idée, non dénuée d'intérêt, d'une contribution de l'agriculture biologique à l'économie, multiplier les approximations qui tendent à affaiblir la valeur du texte et sa portée [Dérades, 2000]. Sur le second point, on admettra qu'en faisant de la Créolité une sorte d'identité matricielle de laquelle procèdent toutes les autres formes d'appartenance, les protagonistes du mouvement prennent le risque de rabattre l'appartenance politique qu'ils revendiquent et jugent légitime sur une identité culturelle qu'ils prétendent, malgré leurs dénégations, authentique [Giraud, 1997].

\footnotetext{
${ }^{10}$ Auxquels il convient d'ajouter Gérard Delver et Bertène Juminer.
} 
De même, les contrariétés actuelles du MIM s'expliquent très largement par l'impossibilité dans laquelle il se trouve d'obtenir une adhésion majoritaire à un projet politique fondé sur une conception de l'identité martiniquaise ramenée, dans sa dimension culturelle, à un corpus de représentations et un système de sens relativement clos sur eux mêmes. Pareille démarche est forcément empreinte d'une forme de culturalisme au point de postuler ou d'escompter un rapport d'extériorité, sur le mode de la causalité univoque, entre culture et action politique et de tenir pour nécessaire une correspondance entre communauté politique et cohérence culturelle [Bayard, op. cit.]. Il en résulte un paradoxe au terme duquel le succès électoral du MIM est d'autant plus important que les chances d'accéder à la souveraineté politique qu'il appelle de ses vœux, et donc d'imposer une identité politique en parfaite congruence avec l'identité culturelle proclamée, semblent s'éloigner. Ce qui le soumet à un dilemme inhérent aux logiques propres à la mécanique démocratique : être confiné, au sein de l'espace politique insulaire, dans un simple rôle de mouvement protestataire ou assumer une banalisation et une dilution de son discours liées à sa participation directe à la gestion quotidienne depuis mars 1998 . L'évolution récente semble d'ailleurs confirmer la prévalence de la deuxième logique à l'œuvre : après avoir longtemps valorisé son étrangeté et cultivé sa particularité au risque de séduire un public restreint dans le cadre de consultations exclusivement locales, le MIM a cherché à élargir son assisse électorale, à conquérir de nouvelles clientèles au point de s'exposer désormais, à travers une gestion réputée prudente de 
l'institution régionale, à une dynamique d'euphémisation de son discours et de sa pratique politique.

Ce n'est donc nullement un hasard si mouvements identitaires et mouvements nationalistes antillais acceptent désormais un horizon politique délimité par la double appartenance à l'ensemble national français et à l'Union européenne. Le champ des possibles en matière d'évolution statutaire n'a pas été simplement borné par l'Etat, ce dernier souscrivant, dans les limites compatibles avec le maintien au sein de la République, à un discours de mise en conformité de la "différence», désormais prise en compte dans l'élaboration et l'application des politiques publiques aux échelons locaux et nationaux [Geisser, 1999] ; il a été également configuré par les porteurs de revendications politico-identitaires, sommés de répondre aux aspirations apparemment contradictoires de sociétés caractérisées par la multiplicité des niveaux d'identification. La traduction politique des attentes exprimées par les populations n'est d'ailleurs pas aisée : elle doit concilier, dans un cadre juridico-institutionnel inédit, l'aspiration universaliste (le principe égalitaire républicain), la prise en compte des particularismes (l'identité culturelle) et la préservation des «droits acquis » dont certains obéissent à une logique purement instrumentale mobilisant alternativement ou simultanément le registre de l'égalitarisme républicain et celui des spécificités locales, entretenant ainsi la dépendance prétendument combattue. D'où cet «étrange paradoxe que celui d'une revendication de particularité qui ne peut produire l'effet de souveraineté recherché par ses 
promoteurs que sous la condition d'un renforcement de ce qu'au moins certains d'entre eux prétendaient ou prétendent encore combattre » [Giraud, 2000: 84]. En somme, le renforcement du pouvoir local qui se dessine à travers la procédure de réforme statutaire prévue par la loi d'orientation pour l'outre mer ne semble nullement remettre en cause le mode de régulation inhérent à la départementalisation. Mieux : il pourrait, même complété par une sphère de compétence législative soigneusement délimitée en amont par le principe d'égalité et le respect des "droits acquis», contribuer à perpétuer ce dernier, et donc la dynamique politico-économique de dépendance qui l'accompagne, tout en faisant formellement et symboliquement disparaître le cadre institutionnel - le département - qui lui était jusqu'alors associé.

\section{Bibliographie}

BAYART Jean-François, 1996, L'illusion identitaire, Paris : Fayard. BERNABE Jean et alii, 1989, Eloge de la créolité, Paris, Gallimard. BERNABE Yves, CAPGRAS Viviane, MURGIER Pascal? (1997), "Les politiques culturelles à la Martinique depuis la décentralisation» in Constant Fred, Daniel Justin, 1946-1996 : Cinquante ans de départementalisation outre mer, Paris, L'Harmattan : 133-151.

BOURDIEU Pierre, «L'opinion publique n'existe pas », Temps modernes, janvier, 318 : 1292-1309.

CONSTANT Fred, 1993, «Les paradoxes de la gestion publique des identités territoriales : à propos des politiques culturelles outre mer", Revue française d'administration publique, $65: 89-99$.

DAHOMAY Jacky, 2000, "Identité culturelle et identité politique. Le cas antillais », Revue de philosophie et de sciences sociales (Comprendre les identités culturelles, sous la dir. De Kymlicka, Mesure Sylvie), $1: 99-118$.

DANIEL Justin, 1997, "L'espace politique martiniquais à l'épreuve de la départementalisation ", in Constant Fred, Daniel Justin, 1946-1996 : Cinquante ans de départementalisation outre mer, Paris, L'Harmattan : 223-259.

DANIEL Justin, 2000a, « Les limites de la démocratie locale en outre mer » in Marion Gérard, Mélanges en hommage à Bernard Vonglis, Paris, L’Harmattan : 85-102. 
DANIEL Justin, 2000b, "Conflits sociaux et construction identitaire à la Martinique », in Bernabé Jean et alii, (2000) Au visiteur lumineux. Des îles créoles aux sociétés plurielles. Mélanges offerts à Jean Benoist, Petit-Bourg : 339-351.

DERADES, 2000, « Le temps des appels. Débat du comité de Rédaction de Dérades le 25 mars $2000 »$, Dérades, 5, 1 semestre : 9-15.

GEISSER Vincent, 1999, «La mise en scène républicaine de l'ethnicité maghrébine : discours d'Etat, discours d'acteurs? in Neveu Catherine (dir.), Espace public et engagement politique. Enjeux et logiques de la citoyenneté locale, Paris, L'Harmattan : 203224.

GIRAUD Michel, 1997, «De la négritude à la créolité : une évolution paradoxale à l'ère départementale », in Constant Fred, Daniel Justin, 1946-1996 : Cinquante ans de départementalisation outre mer, Paris, L'Harmattan : 373-403.

GIRAUD Michel, 2000, "Après la colonie, la nation? Le devenir politique des départements français d'Amérique en question », Pouvoirs dans la Caraïbe, 12.

HERMET Guy, 2001, Les populismes dans le monde, Paris, Fayard.

JULES-ROSETTE Bennneta, MARTIN Denis-Constant, 1997, «Cultures populaires, identités et politiques ", Les Cahiers du CERI, n 17.

LEVI-STRAUSS Claude, 1977, L'identité, Séminaire interdisciplinaire dirigé par LéviStrauss, professeur au collège de France, Paris, Grasset.

LISE Claude, 2001, Allocution du Sénateur Claude LISE lors du débat sur l'Avenir de l'Europe organisé par le Préfet, 27 octobre, Madiana.

MANIFESTE, 2000, «Débat sur le changement de statut: le triomphe de l'autonomie », France-Antilles, 11 mars.

MARTIN Denis-Constant, 1994, Cartes d'identité. Comment dit-on "nous" en politique, Paris, Presses de la Fondation Nationale des Sciences Politiques.

NEVEU Catherine, 1999, "Présentation » in Neveu Catherine (dir.), Espace public et engagement politique. Enjeux et logiques de la citoyenneté locale, Paris, L'Harmattan : 9-13. PROJET MARTINIQUE, 2000, Conférence de Martinique, 2 décembre, Madiana.

ROSANVALLON Pierre, 2000, La démocratie inachevée. Histoire de la souveraineté du peuple en France, Paris, Gallimard.

SUVELOR Roland, 1983, «Eléments historiques pour une approche socioculturelle », Les Temps Modernes, 39(441-442) : 2174-2208.

TABOA Leonetti, 1990, "Stratégies identitaires et minorités : le point de vue du sociologue », in Camillieri, Carmel et alii., 1990, Stratégies identitaires, Paris, PUF : 4383.

TRESOR Georges, 1999, «Penser la question politique en Guadeloupe », Dérades, 4, 2e semestre : 25-39. 
Annexe 1

La redécouverte de l'identité à travers les musées :

L'exemple de l'écomusée de Martinique

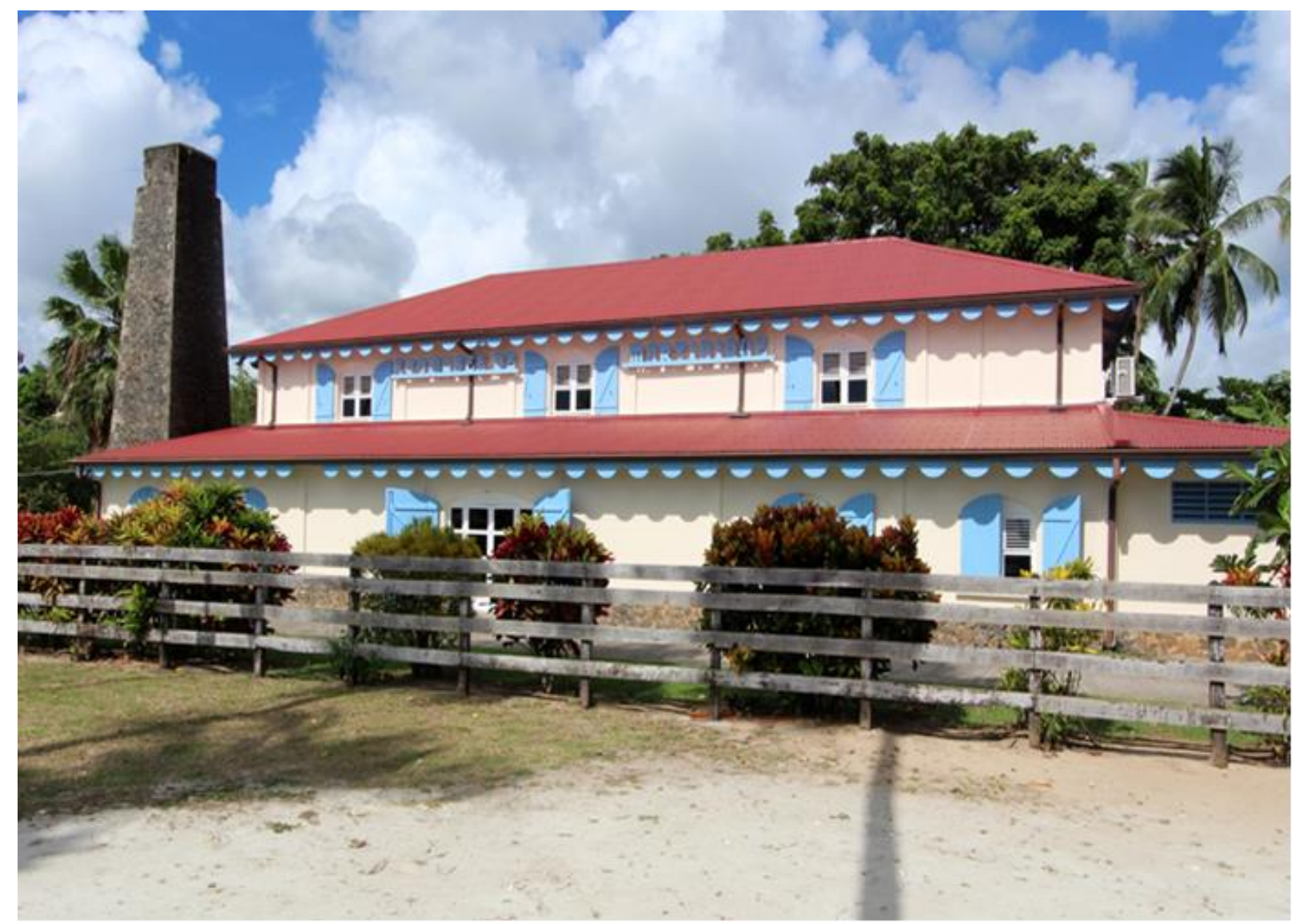

L'écomusée de la Martinique a vu le jour grâce à la volonté et à l'action de l'Association martiniquaise pour la promotion des arts et traditions populaires (AMPATP) présidée par Louis-Félix Ozier-Lafontaine. Symboliquement situé dans une ancienne distillerie et sur un site - protégé - comportant des vestiges amérindiens, il devient musée régional en 1992. L'écomusée invite à se plonger dans la mémoire collective, de la période amérindienne au gouvernement de Vichy, grâce à diverses évocations. On y trouve, par exemple, la reconstitution fidèle d'un intérieur créole entre 1930 et 1950, des panneaux illustrant l'histoire de la Martinique et faisant revivre des civilisations disparues ainsi que des périodes douloureuses de cette histoire. Dès sa création, l'écomusée s'est vu assigner comme objectif de conforter l'identité martiniquaise. 
Une chanson populaire sur les menaces que représente le «loup Europe »

\section{- "Le Gran Méchan Lou»(Djo Dézormo)}

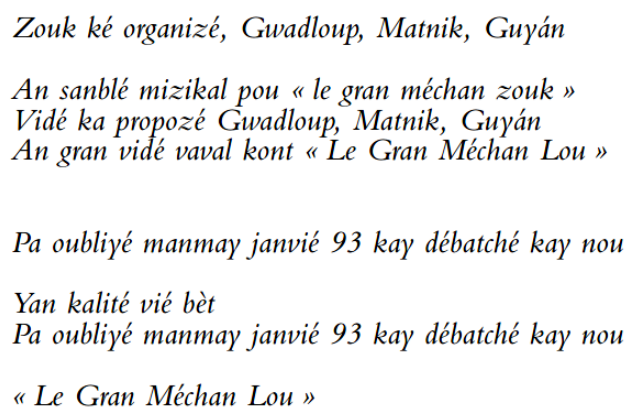

Houhou voici le lou (ter), le lou ki lé dévoré nou Houhou voici le lou (ter), le lou ki lé èstentjé nou

[Bêêêe... mach (ter), mach] (× 4)] (bis)

Kay nifê $(\times 12)$

Houhou voici le lou (ter), le lou ki lé dévoré nou...

Pourquoi t'as de grands yeux comme ça

Pourquoi de grands cheveux comme ça

Pourquoi t'as une longue queue comme ça

Pourquoi t'as de grandes dents comme ça

[Pour te croquer $(\times 4)$, te dévorer] (bis)

Houhou voici le lou (ter), le lou ki lé dévoré nou...

Erèzdibonnè nou ni kat dépité, kat dépité fransé, fransé-matinitjè

Athos, Porthos, Aramis, d'Artagnan (× 4)

Erèzdibonnè nou ni lé dé konsèy

Konsèy Jénéral, Konsèy Réjional

Twôp konséyé pa bon, lé konséyè pa lé péyè $(\times 4)$

Houhou voici le lou (ter), le lou ki lé dévoré nou...
En Martinique, Guadeloupe et Guyane de grands zouks seront organisés

Un rassemblement musical pour "le grand méchant zouk " Le Carnaval propose en Guadeloupe, Martinique et Guyane Un grand "vidée " de carnaval contre "le Grand Méchant Loup "

N'oublions pas mes amis qu'en janvier 93 débarquera chez nous

Une drôle de vilaine bête

N'oublions pas mes amis qu'en janvier 93 débarquera chez nous

"Le Grand Méchant Loup "

Hou ! hou ! voici le loup (ter), le loup qui veut nous dévorer Hou! hou! voici le loup (ter), le loup qui veut nous esquinter

[Bêêê̂... ouste! (ter), ouste] $(\times 4)$ (bis)

On en bavera $(\times 12)$

Hou ! hou ! voici le loup (ter), le loup qui veut nous dévorer...

Pourquoi t'as de grands yeux comme ça

Pourquoi de grands cheveux comme ça

Pourquoi t'as une longue queue comme ça

Pourquoi t'as de grandes dents comme ça

[Pour te croquer $(\times 4)$, te dévorer] (bis)

Hou! hou! voici le loup (ter), le loup qui veut nous dévorer...

Fort heureusement nous avons quatre députés, quatre députés français, français-martiniquais

Athos, Porthos, Aramis, d'Artagnan (× 4)

Fort heureusement nous avons deux conseils,

Le conseil général, le conseil régional.

Trop de conseillers, ce n'est pas bon : les conseillers ne sont pas les payeurs $(\times 4)$

Hou ! hou! voici le loup (ter), le loup qui veut nous dévorer... 